\title{
A study on the onset of thermally modulated Darcy-Bénard convection
}

\author{
Om P. Suthar • P. G. Siddheshwar • \\ B. S. Bhadauria
}

Received: 31 March 2015 / Accepted: 4 March 2016

(C) Springer Science+Business Media Dordrecht 2016

\begin{abstract}
A stability analysis of linearized Rayleigh-Bénard convection in a densely packed porous layer was performed using a matrix differential operator theory. The boundary temperatures were assumed to vary periodically with time in a sinusoidal manner. The correction in the critical Darcy-Rayleigh number was computed and depicted graphically. It was shown that the phase difference between the boundary temperatures rather than the frequency of modulated temperatures decides the nature of influence of modulation on the onset of convection. Conclusions were drawn regarding the possible transitions from harmonic to subharmonic solutions. The results on the onset of thermally modulated convection in a rectangular porous enclosure were obtained using those on the modulated Darcy-Bénard convection.
\end{abstract}

Keywords Darcy-Bénard convection - Matrix differential operator - Rectangular enclosure $\cdot$ Subharmonic instability $\cdot$ Temperature modulation

Mathematics Subject Classification $76 \mathrm{E} 06 \cdot 76 \mathrm{E} 15 \cdot 76 \mathrm{M} 45 \cdot 76 \mathrm{R} 10 \cdot 76 \mathrm{~S} 05$

\section{Introduction}

Natural convection can occur in a fluid-saturated porous medium if the buoyancy forces within the fluid are sufficiently strong to overcome viscous drag. The classical Horton-Rogers-Lapwood problem [1,2] considers an infinite horizontal porous layer with the lower surface maintained at a higher temperature than that of the upper surface (Fig. 1a). They found that convection occurred if the dimensionless Darcy-Rayleigh number (defined later in the paper) exceeds the critical value of $4 \pi^{2}$. Following on from this, results are found for the time-independent boundary conditions of a Dirichlet or Neumann or Robin type [3,4]. An additional small-amplitude temperature modulation

O. P. Suthar $(\varangle)$

Department of Mathematics, School of Advanced Sciences, VIT University, Vellore 632014, India

e-mail: ompsuthar@gmail.com; ompsuthar@vit.ac.in

P. G. Siddheshwar

Department of Mathematics, Bangalore University, Jnanabharathi Campus, Bangalore 560 056, India

B. S. Bhadauria

Department of Mathematics, Institute of Science, Banaras Hindu University, Varanasi 221005, India 
(a)

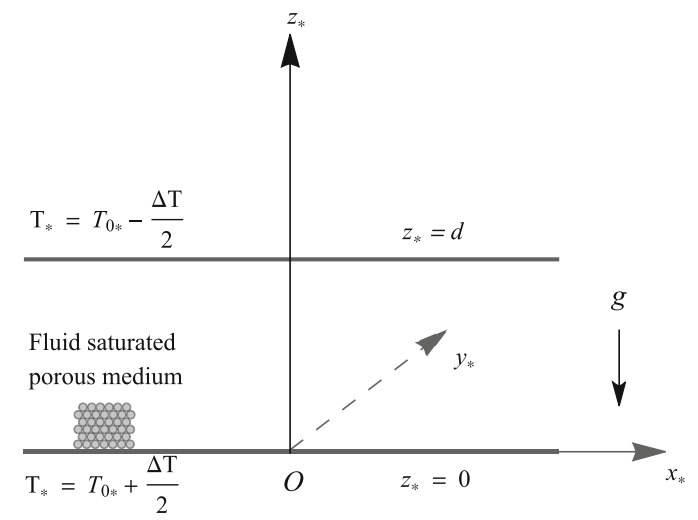

(b)

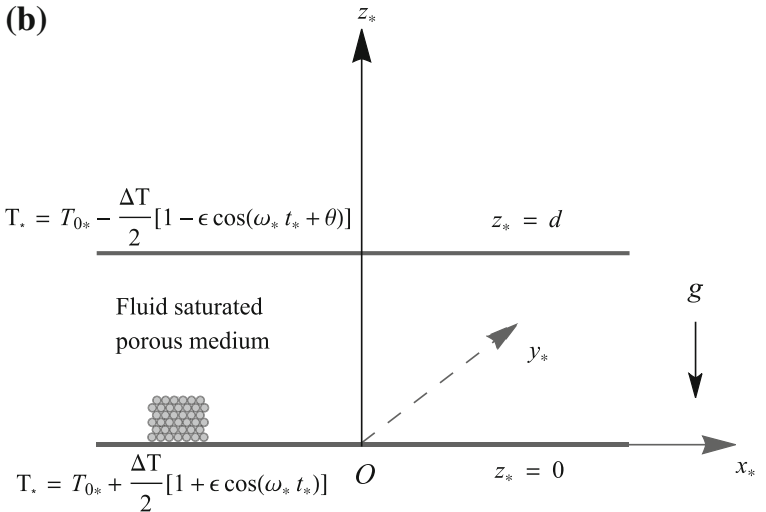

Fig. 1 Schematic of Darcy-Bénard convection $\mathbf{a}$ without modulation and $\mathbf{b}$ with modulation

(time-periodic boundary temperature) as considered in the Darcy-Bénard problem here necessarily means that the $4 \pi^{2}$ for the critical eigenvalue needs to have a correction with a contribution from the temperature modulation (see [5-11]). Experiments on modulated Rayleigh-Bénard convection were reported by Niemela and Donnelly [12]. Their results showed that the possibility of subcritical or supercritical instability depends on the type of modulation. These results on the temperature modulation of convection are practically quite important in the sense that they may be effectively used to regulate convection - either enable or disable convection. This aspect of modulation is one of the motivating factors for our work.

The critical Darcy-Rayleigh number in the Lapwood problem was obtained by solving for the eigenvalue of a homogeneous boundary value problem using classical methods like Galerkin or variants of the shooting method. These procedures work for constant boundary conditions. Of concern in this paper is the Darcy-Bénard problem with time-periodic boundary conditions, and the boundary value eigenproblem arising here will have to be solved using the Venezian approach or Floquet theory or by the matrix differential operator method. The Venezian approach reduces the system of governing equations to a single equation of higher order in temperature by eliminating the velocity [6-11]. If the boundary conditions are other than stress free and isothermal, then additional boundary conditions required by the higher-order equation are difficult to obtain and difficult to handle. The matrix differential operator method retains the system of governing equations as it is and does not require the generation of extra boundary conditions. Hence we feel that the matrix differential operator method is preferable over the Venezian approach because of its ease of use. It is appropriate to mention here that, alternatively, one could use Floquet theory, which is based on the fact that the governing equations for modulated problems are to be written down in terms of a nonautonomous Mathieu-type equation [13]. The current problem can be reduced to a form suitable for Floquet theory, but the authors feel it is advantageous to use the equations as they are, and that approach has been followed in the matrix differential operator method.

We present some new results on temperature modulation whose stabilizing or destabilizing effect is dictated by both frequency and phase difference rather than by frequency only. The perturbation expansion employed in this paper incorporates the general frequency response of the system for the modulated forcing, which enables us to study the transitions from harmonic to subharmonic solutions. It is shown in the paper that the results of the corresponding problem of thermally modulated convection in a rectangular porous enclosure can be obtained easily from the results of the present study. The nonmodulated thermal convection in a porous enclosure was first reported by Beck [14]. The reported results for the nonmodulated problem state that the critical Rayleigh number for the onset of stationary convection is always greater than or equal to the critical Rayleigh number for Darcy-Bénard convection. There is no such result available in regard to thermally modulated convection in a fluid-saturated rectangular porous enclosure. A comprehensive review of the topic has been given by Nield and Bejan [15, Chap. 6] and Tyvand [16, Chap. 4]. We now proceed with the formulation of Darcy-Bénard convection with temperature modulation. 


\section{Problem formulation}

The classical setup of Darcy-Bénard convection in a fluid-saturated porous layer [15, Chap. 6] is considered here with an additional small-amplitude temperature modulation as shown in Fig. 1b. The system is represented by the following governing equations:

Conservation of mass:

$\nabla_{*} \cdot \mathbf{q}_{*}=0$,

Conservation of momentum:

$\frac{1}{\phi} \frac{\partial \mathbf{q}_{*}}{\partial t_{*}}=-\frac{1}{\rho_{0 *}} \nabla_{*} p_{*}+\frac{\rho_{*}}{\rho_{0 *}} \mathbf{g}-\frac{v}{K} \mathbf{q}_{*}$,

Conservation of energy:

$\frac{\partial T_{*}}{\partial t_{*}}+\frac{1}{\phi}\left(\mathbf{q}_{*} \cdot \nabla_{*}\right) T_{*}=\kappa \nabla_{*}^{2} T_{*}$,

Equation of state:

$\rho_{*}=\rho_{0 *}\left[1-\beta\left(T_{*}-T_{0 *}\right)\right]$,

where $\mathbf{q}_{*}, \rho_{0 *}, \rho_{*}, p_{*}, T_{0 *}$, and $T_{*}$ are the seepage velocity, reference fluid density, fluid density, fluid pressure, reference fluid temperature, and fluid temperature, respectively, at some point $\left(x_{*}, y_{*}, z_{*}\right)$ in the system at some time $t_{*} ; \mathbf{g}=(0,0,-g)$ is the acceleration due to the gravitational force; $\phi$ is the porosity of the porous medium; $v$ is the kinematic viscosity; $K$ is the permeability; and $\kappa$ is the effective thermal diffusivity of the system. The asterisk $(*)$ indicates that the quantities are dimensional.

The externally imposed time-periodic boundary temperatures are assumed in the following form [5]:

$T_{*}= \begin{cases}T_{0 *}+\frac{\Delta T}{2}\left[1+\epsilon \cos \left(\omega_{*} t_{*}\right)\right] & \text { at } z_{*}=0, \\ T_{0 *}-\frac{\Delta T}{2}\left[1-\epsilon \cos \left(\omega_{*} t_{*}+\theta\right)\right] & \text { at } z_{*}=d,\end{cases}$

where $\epsilon$ is the small amplitude of modulation, $\omega_{*}$ is the dimensional modulation frequency, and $\theta$ is the phase difference. The governing equations are rendered dimensionless using the following scaling:

$t_{*}=\frac{d^{2}}{\kappa} t, \quad x_{*}=\mathrm{d} x, \quad T_{*}=\Delta T T, \quad \mathbf{q}_{*}=\frac{\phi \kappa}{d} \mathbf{q}$,

$\omega_{*}=\frac{\kappa}{d^{2}} \omega, \quad p_{*}=\frac{\phi \rho_{0} \nu \kappa}{K} p, \quad \rho=\frac{\rho_{*}-\rho_{0 *}}{\rho_{0 *}}$.

Equations (1)-(3) in their dimensionless form read

$\nabla \cdot \mathbf{q}=0$,

$\frac{1}{\mathrm{Va}} \frac{\partial \mathbf{q}}{\partial t}=-\nabla p+\mathrm{Ra} T-\mathbf{q}$,

$\frac{\partial T}{\partial t}+(\mathbf{q} \cdot \nabla) T=\nabla^{2} T$.

In the preceding equations, the dimensionless parameters are Vadasz number (Va) and Darcy-Rayleigh number (Ra) given by

$\mathrm{Va}=\frac{\phi \operatorname{Pr}}{\mathrm{Da}}, \quad \operatorname{Pr}=\frac{\nu}{\kappa}, \quad \mathrm{Da}=\frac{K}{d^{2}}, \quad \mathrm{Ra}=\frac{\beta \Delta T \mathrm{~g} K d}{\phi \nu \kappa}$. 
The basic state is assumed to be quiescent, and the quantities in this state are given by

$\mathbf{q}_{\mathbf{b}}=(0,0,0), \quad \rho=\rho_{\mathrm{b}}(z, t), \quad p=p_{\mathrm{b}}(z, t), \quad T=T_{\mathrm{b}}(z, t)$.

These basic state quantities satisfy the following equations:

$\frac{\partial p_{\mathrm{b}}}{\partial z}=\operatorname{Ra} T_{\mathrm{b}}$

$\frac{\partial T_{\mathrm{b}}}{\partial t}=\frac{\partial^{2} T_{\mathrm{b}}}{\partial z^{2}}$.

The solution of Eq. (10), subject to the non-dimensional form of the boundary conditions (5), is given by

$T_{\mathrm{b}}(z, t)=\frac{1}{2}(1-2 z)+\epsilon F(z, t)$,

where

$F(z, t)=\frac{1}{2}[f(z) \exp (\mathrm{i} \omega t)+\bar{f}(z) \exp (-\mathrm{i} \omega t)]$,

$f(z)=A(\lambda) \mathrm{e}^{\lambda z}+A(-\lambda) \mathrm{e}^{-\lambda z}$,

$A(\lambda)=\frac{1}{2} \frac{\left(\mathrm{e}^{-\mathrm{i} \theta}-\mathrm{e}^{-\lambda}\right)}{\left(\mathrm{e}^{\lambda}-\mathrm{e}^{-\lambda}\right)}, \quad \lambda=(1-\mathrm{i}) \sqrt{\frac{\omega}{2}}$,

and a bar over a quantity indicates its conjugate.

The perturbations on the basic state are superposed in the form

$\mathbf{q}=\mathbf{q}_{\mathbf{b}}+\mathbf{q}^{\prime} ; \quad p=p_{\mathrm{b}}+p^{\prime} ; \quad T=T_{\mathrm{b}}+T^{\prime} ; \quad \rho=\rho_{\mathrm{b}}+\rho^{\prime}$,

where the perturbations are of infinitesimal amplitude. Substituting Eq. (14) into Eqs. (6)-(8) and using Eqs. (9) and (10) in the resulting equations, the following equations are arrived at:

$\nabla \cdot \mathbf{q}^{\prime}=0$

$\frac{1}{\mathrm{Va}} \frac{\partial \mathbf{q}^{\prime}}{\partial t}=-\nabla p^{\prime}+\operatorname{Ra} T^{\prime}-\mathbf{q}^{\prime}$,

$\frac{\partial T^{\prime}}{\partial t}+\left(\mathbf{q}^{\prime} \cdot \nabla\right) T^{\prime}+w^{\prime} \frac{\partial T_{\mathrm{b}}}{\partial z}=\nabla^{2} T^{\prime}$.

Only two-dimensional disturbances are considered, and the stream function $\psi^{\prime}$ is defined as

$\left(u^{\prime}, v^{\prime}, w^{\prime}\right)=\left(\frac{\partial \psi^{\prime}}{\partial z}, 0,-\frac{\partial \psi^{\prime}}{\partial x}\right)$

Eliminating the pressure term from Eq. (16) and then introducing the stream function yields the following equation:

$\frac{1}{\mathrm{Va}} \frac{\partial}{\partial t}\left(\nabla^{2} \psi^{\prime}\right)=-\nabla^{2} \psi^{\prime}-\operatorname{Ra} \frac{\partial T^{\prime}}{\partial x}$. 
On substituting Eq. (18) into Eq. (17), the heat transport equation is obtained in the form

$\frac{\partial T^{\prime}}{\partial t}-\frac{\partial\left(\psi^{\prime}, T^{\prime}\right)}{\partial(x, z)}=\nabla^{2} T^{\prime}+\frac{\partial \psi^{\prime}}{\partial x} \frac{\partial T_{\mathrm{b}}}{\partial z}$

where $\nabla^{2} \equiv \frac{\partial^{2}}{\partial x^{2}}+\frac{\partial^{2}}{\partial z^{2}}$.

The boundary conditions for the perturbed quantities are

$$
\left.\begin{array}{ll}
\psi^{\prime}=0 & \text { at } z=0,1 \\
T^{\prime}=0 & \text { at } z=0,1
\end{array}\right\}
$$

Note that the basic state solution influences the stability problem through the factor $\partial T_{\mathrm{b}} / \partial z$ in Eq. (20), which is given by

$\frac{\partial T_{\mathrm{b}}}{\partial z}(z, t)=-1+\frac{\epsilon}{2}\left[f_{1}(z) \exp (\mathrm{i} \omega t)+\bar{f}_{1}(z) \exp (-\mathrm{i} \omega t)\right]$,

where

$f_{1}(z)=A_{1}(\lambda) \mathrm{e}^{\lambda z}+A_{1}(-\lambda) \mathrm{e}^{-\lambda z}$,

$A_{1}(\lambda)=\frac{\lambda}{2} \frac{\left(\mathrm{e}^{-\mathrm{i} \theta}-\mathrm{e}^{-\lambda}\right)}{\left(\mathrm{e}^{\lambda}-\mathrm{e}^{-\lambda}\right)}, \quad \lambda=(1-\mathrm{i}) \sqrt{\frac{\omega}{2}}$.

In the next section a linear stability analysis of system (19)-(20) is performed to arrive at an analytical expression for the eigenvalue of the nonmodulated problem and the correction to it dictated by the modulation effect.

\section{Linear stability analysis}

To perform a linear stability analysis, we omit the nonlinear term in Eq. (20) and make the following assumptions:

$$
\left(\begin{array}{l}
\psi^{\prime}(x, z, t) \\
T^{\prime}(x, z, t)
\end{array}\right)=\left(\begin{array}{c}
\Psi(z, t) \sin (k x) \\
\Theta(z, t) \cos (k x)
\end{array}\right) .
$$

From solution (24) it is obvious that we are looking for periodic solutions with wave number $k$ in the $x$-direction. The governing equations for linear stability are then given by

$$
\begin{aligned}
& {\left[\frac{1}{\mathrm{Va}} \frac{\partial}{\partial t}+1\right]\left(D^{2}-k^{2}\right) \Psi=k_{\mathrm{c}} \operatorname{Ra} \Theta,} \\
& {\left[\frac{\partial}{\partial t}-\left(D^{2}-k^{2}\right)\right] \Theta=k_{\mathrm{c}} \Psi D T_{\mathrm{b}},}
\end{aligned}
$$

where $D \equiv \frac{\partial}{\partial z}$. A scaled temperature $\Gamma=k_{\mathrm{c}} \operatorname{Ra} \Theta$ is now used to rewrite the governing equations (25)-(26) in the form

$$
\left[\frac{1}{\operatorname{Va}} \frac{\partial}{\partial t}+1\right]\left(D^{2}-k^{2}\right) \Psi=\Gamma,
$$




$$
\left[\frac{\partial}{\partial t}-\left(D^{2}-k^{2}\right)\right] \Gamma=k^{2} \operatorname{Ra} \Psi D T_{\mathrm{b}} .
$$

A hybrid approach between that of Raju and Bhattacharya [17] and Siddheshwar [18] is followed, and an assumption is made about the following expansions for $\Psi, \Gamma$, and Ra using the small-modulation amplitude $\epsilon$ as an expansion parameter:

$$
\begin{aligned}
& \Psi(z, t)=\Psi_{\mathrm{S}}(z)+\frac{1}{2}\left[\Psi_{1}(z) \mathrm{e}^{\mathrm{i} q t}+\tilde{\Psi}_{1}(z) \mathrm{e}^{-\mathrm{i} q t}\right], \\
& \Gamma(z, t)=\Gamma_{\mathrm{S}}(z)+\frac{1}{2}\left[\Gamma_{1}(z) \mathrm{e}^{\mathrm{i} q t}+\bar{\Gamma}_{1}(z) \mathrm{e}^{-\mathrm{i} q t}\right], \\
& \mathrm{Ra}=\mathrm{Ra}^{(0)}+\epsilon^{2} \mathrm{Ra}^{(2)}+\cdots,
\end{aligned}
$$

where

$$
\Psi_{\mathrm{s}}(z)=\Psi_{\mathrm{s}}^{(0)}+\epsilon^{2} \Psi_{\mathrm{s}}^{(2)}+\cdots, \quad \Gamma_{\mathrm{s}}(z)=\Gamma_{\mathrm{s}}^{(0)}+\epsilon^{2} \Gamma_{\mathrm{s}}^{(2)}+\cdots, \quad \Psi_{1}=\epsilon \Psi_{1}^{(1)}+\cdots, \quad \Gamma_{1}=\epsilon \Gamma_{1}^{(1)}+\cdots .
$$

In the preceding expressions, $q$ represents the general frequency response of the system for the modulated temperature forcing. At this juncture we note that the $D T_{\mathrm{b}}$ term has frequency $\omega$, which is multiplied by $\psi$. This product of $D T_{\mathrm{b}}$ and $\psi$ might cause a cascade of frequencies. Now, more importantly, recognizing that it is densely packed porous media that we are considering, it becomes clear that, as in the case of other convection problems using the Darcy model, $q=\omega$ is the dominant harmonic, and integral multiples of $\omega$ would not lead to significant changes in the conclusions that have been made. This is because densely packed porous media provide a much smaller window for the manifestation of any type of instability and in fact damps whatever instabilities do manifest. Further, regarding the subharmonics, we have made allowance for the subharmonic responses of various orders, and integral multiples of these give us the harmonics only. Dominant harmonics and a few subharmonics were therefore decided upon for investigation. The value $q=\omega$ corresponds to a harmonic response of the system, whereas $q=\omega / 2, \omega / 3, \ldots$ correspond to subharmonic responses of order $1 / 2,1 / 3, \ldots[19,20]$. We have considered the subharmonic of order $1 / 2$ only in the calculations and have reported the results. The subharmonics of other orders can also be obtained easily. Analysis of such a result was in fact conducted, and the conclusion was similar to the representative subharmonic of order $1 / 2$. Given the fact that the exercise in the problem is one of estimating the eigenvalue with correction due to modulation, the choice of the harmonic $q=\omega$ and subharmonics of various orders seemed to be the correct approach.

As per the observation of Venezian [5], the critical wave number of the nonmodulated and the modulated systems may be taken to be the same. In view of this, a perturbation expansion for the wave number is not chosen.

Substituting Eqs. (29)-(31) into Eqs. (27)-(28) and equating the coefficients of various powers of $\epsilon$ now leads to the following systems:

\section{Zeroth order}

$$
\left(\begin{array}{cc}
D^{2}-k^{2} & -1 \\
-k^{2} \mathrm{Ra}^{(0)} & D^{2}-k^{2}
\end{array}\right)\left(\begin{array}{c}
\Psi_{\mathrm{s}}^{(0)} \\
\Gamma_{\mathrm{s}}^{(0)}
\end{array}\right)=\left(\begin{array}{l}
0 \\
0
\end{array}\right),
$$

$\psi_{\mathrm{s}}^{(0)}=\Gamma_{\mathrm{s}}^{(0)}=0 \quad$ at $z=0,1$;

\section{First order}

$$
\mathcal{A}\left(\begin{array}{c}
\Psi_{1}^{(1)} \\
\Gamma_{1}^{(1)}
\end{array}\right)+f(q) \overline{\mathcal{A}}\left(\begin{array}{l}
\bar{\Psi}_{1}^{(1)} \\
\bar{\Gamma}_{1}^{(1)}
\end{array}\right)=\left(\begin{array}{l}
0 \\
\mathcal{R}
\end{array}\right),
$$


$\Psi_{1}^{(1)}=\Gamma_{1}^{(1)}=0$ at $z=0,1$,

where

$\mathcal{A}=\left(\begin{array}{cc}\left(1+\frac{\mathrm{i} q}{\mathrm{Va}}\right)\left(D^{2}-k^{2}\right) & -1 \\ -k^{2} \mathrm{Ra}^{(0)} & \left(D^{2}-k^{2}-\mathrm{i} q\right)\end{array}\right)$,

$\mathcal{R}=\left(f_{1}(z) h(q)+\bar{f}_{1}(z) g(q)\right) \Psi_{\mathrm{s}}^{(0)}, f(q)=\left\langle\mathrm{e}^{-\mathrm{i} q t}, \mathrm{e}^{-\mathrm{i} q t}\right\rangle, g(q)=\left\langle\mathrm{e}^{-\mathrm{i} q t}, \mathrm{e}^{-\mathrm{i} \omega t}\right\rangle, h(q)=\left\langle\mathrm{e}^{-\mathrm{i} q t}, \mathrm{e}^{\mathrm{i} \omega t}\right\rangle$, and $\langle\cdot, \cdot\rangle$ represents time average over one modulation period, i.e., over the interval $[0,2 \pi / \omega]$;

Second order

$$
\left(\begin{array}{cc}
D^{2}-k^{2} & -1 \\
-k^{2} \operatorname{Ra}^{(0)} & D^{2}-k^{2}
\end{array}\right)\left(\begin{array}{c}
\Psi_{\mathrm{s}}^{(2)} \\
\Gamma_{\mathrm{s}}^{(2)}
\end{array}\right)=\left(\begin{array}{c}
\mathcal{R}_{1} \\
\mathcal{R}_{2}
\end{array}\right)
$$

where $\mathcal{R}_{1}=0$ and

$$
\begin{aligned}
& \mathcal{R}_{2}=k^{2} \operatorname{Ra}_{\mathrm{c}}^{(2)} \Psi_{\mathrm{s}}^{(0)}-\frac{k^{2}}{4} \mathrm{Ra}^{(0)}\left[\Psi_{1}^{(1)} f_{1}(z) \bar{g}(q)+\bar{\Psi}_{1}^{(1)} \bar{f}_{1}(z) g(q)+\Psi_{1}^{(1)} \bar{f}_{1}(z) \bar{h}(q)+\bar{\Psi}_{1}^{(1)} f_{1}(z) h(q)\right], \\
& \Psi_{\mathrm{s}}^{(2)}=\Gamma_{\mathrm{s}}^{(2)}=0 \quad \text { at } \quad z=0,1 .
\end{aligned}
$$

The solution of the zeroth-order system is given by

$\Psi_{\mathrm{s}}^{(0)}=A \sin (\pi z), \quad \mathrm{Ra}^{(0)}=\frac{\left(\pi^{2}+k^{2}\right)^{2}}{k^{2}}$.

It can easily be shown that $k_{\mathrm{c}}=\pi$ and, hence, $\mathrm{Ra}_{\mathrm{c}}^{(0)}=4 \pi^{2}$. These are the critical values of the wave number and Rayleigh number of the nonmodulated Darcy-Bénard system.

Because the secular terms need to be removed from the right-hand side of Eq. (35), we use the standard Fredholm solvability condition, which requires that the right-hand side lie in the kernel of the operator. Applying the solvability condition $[18,21,22]$ to the second-order system (35), the correction Rayleigh number $\mathrm{Ra}_{\mathrm{c}}^{(2)}$ can be, on rearrangement, obtained in the form

$\operatorname{Ra}_{\mathrm{c}}^{(2)}=\left[\frac{\int_{0}^{1} \tilde{\Gamma}_{\mathrm{s}}^{(0)}\left\{\Psi_{1}^{(1)} f_{1}(z) \bar{g}(q)+\bar{\Psi}_{1}^{(1)} \bar{f}_{1}(z) g(q)+\Psi_{1}^{(1)} \bar{f}_{1}(z) \bar{h}(q)+\bar{\Psi}_{1}^{(1)} f_{1}(z) h(q)\right\} \mathrm{d} z}{\int_{0}^{1} \bar{\Gamma}_{\mathrm{s}}^{(0)} \Psi_{\mathrm{s}}^{(0)} \mathrm{d} z}\right] \pi^{2}$,

where $\tilde{\Gamma}_{\mathrm{s}}^{(0)}$ is the solution of the adjoint problem of the zeroth-order system (32). It can be observed that to calculate the correction in the critical Rayleigh number, we need to obtain the first-order solutions. Before attempting to solve Eqs. (33)-(34), we note that $f(\omega)$ and $f(\omega / 2)$ are zero, which makes it easier for us to solve the first-order system. Thus, henceforth we restrict our attention to the cases where $q=\omega$ (harmonic) or $q=\omega / 2$ (subharmonic of order $1 / 2$ ). Now with this restriction we can eliminate $\Gamma_{1}^{(1)}$ from system (33) to obtain

$$
\left[\left\{\frac{\mathrm{i} q}{\mathrm{Va}}\left(D^{2}-k_{\mathrm{c}}^{2}\right)+\left(D^{2}-k_{\mathrm{c}}^{2}\right)\right\}\left(D^{2}-k_{\mathrm{c}}^{2}-\mathrm{i} q\right)-k_{\mathrm{c}}^{2} \operatorname{Ra}_{\mathrm{c}}^{(0)}\right] \Psi_{1}^{(1)}=-k_{\mathrm{c}}^{2} \operatorname{Ra}_{\mathrm{c}}^{(0)} \Psi_{\mathrm{s}}^{(0)}\left[f_{1}(z) h(q)+\bar{f}_{1}(z) g(q)\right] .
$$

The boundary conditions for solving Eq. (40) are

$\Psi_{1}^{(1)}=D^{2} \Psi_{1}^{(1)}=0 \quad$ at $\quad z=0,1$, 
where the condition $D^{2} \Psi_{1}^{(1)}=0$ was obtained from Eq. (33). The solution for the fourth-order differential equation (40) can be readily obtained numerically using the built-in function NDSolve in Mathematica 9.0.

Note that the same amplitude $A$ appears in both $\Psi_{\mathrm{s}}^{(0)}$ and $\Psi_{1}^{(1)}$. Because $\Psi_{1}^{(1)}$ was obtained numerically, the built-in function NIntegrate in Mathematica 9.0 is used in evaluating $\operatorname{Ra}_{\mathrm{c}}^{(2)}$.

We now move on to investigate the onset of two-dimensional modulated convection in a rectangular porous enclosure. It should be noted here that the governing equations for the Darcy-Bénard and porous enclosure problems are the same; what differs are the boundary conditions, which are discussed in detail in the following section.

\section{Temperature modulation of two-dimensional convection of a Newtonian fluid in a rectangular porous enclosure}

In this case the region of interest is

$\{(x, z): 0 \leq x \leq b, 0 \leq z \leq 1\}$,

where $b$ is the aspect ratio (nondimensional width) of the rectangular enclosure. The conditions imposed on the walls of the rectangular enclosure are

$\left.\begin{array}{lll}\psi^{\prime}=T^{\prime}=0 & \text { at } z=0 \text { and } 1, & \forall x \in(0, b), \\ \psi^{\prime}=\frac{\partial T^{\prime}}{\partial x}=0 & \text { at } x=0 \text { and } b, & \forall z \in(0,1) .\end{array}\right\}$

The stream function for this two-dimensional convective flow is given by (see Tyvand [16, Chap. 4])

$\psi^{\prime}=A \sin \left(\frac{n \pi x}{b}\right) \sin (\pi z)$

An analysis similar to that presented in the previous section leads to exactly the same zeroth-, first-, and second-order systems. The critical Darcy-Rayleigh number for the nonmodulated rectangular porous enclosure problem can be readily obtained, using Eq. (43) in Eq. (32), as

$\operatorname{Ra}_{\mathrm{c}}^{(0)}=\pi^{2} \min _{n \in \mathbf{N}}\left(\frac{n}{b}+\frac{b}{n}\right)^{2}$,

where $\mathbf{N}$ represents the set of all natural numbers. In the next section we discuss in detail the results obtained for the modulated Darcy-Bénard and modulated rectangular enclosure problems.

\section{Results and discussion}

The Darcy-Bénard problem is taken up for investigation under the effect of time-varying boundary temperatures. The problem is tackled analytically by assuming the modulation to be of small amplitude. In the present study two types of temperature modulations are considered:

1. Synchronous temperature modulation, in which case $\theta=0$;

2. Asynchronous temperature modulation, in which case $\theta \neq 0$.

The assumption of a small-amplitude modulation allows us to utilize the amplitude of modulation as a perturbation parameter in expanding the system quantities in series form. Such an expansion results in systems of different orders, which are dealt with by using matrix differential operator theory. The lowest-order system yields as its eigenvalue the stationary Rayleigh number in the absence of modulation. The solution of the first-order system is obtained in order to calculate the eigenvalue of the third-order system. It can be observed from Eqs. (35) and (36) that 

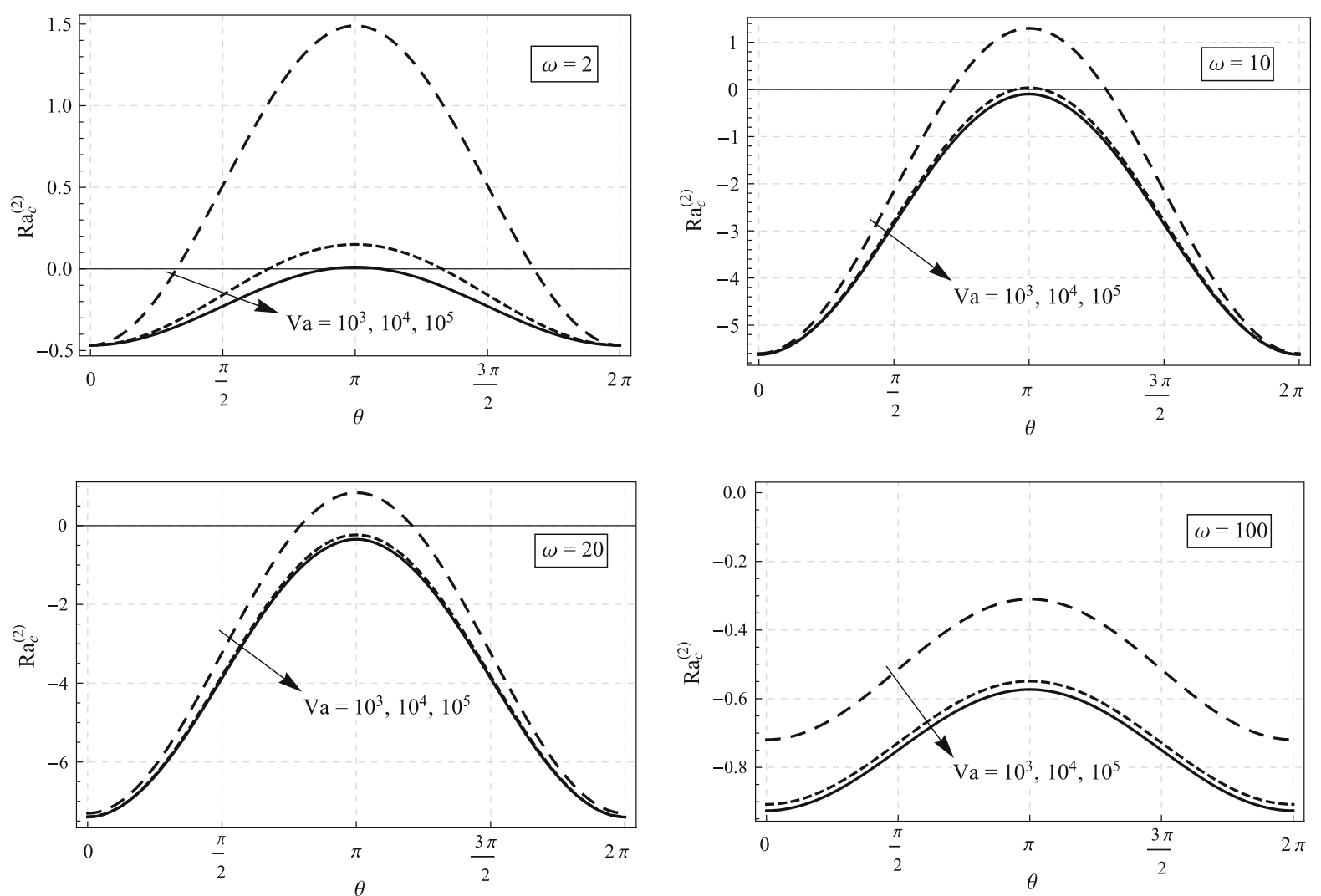

Fig. 2 Effect of phase difference $\theta$ on correction Rayleigh number $\operatorname{Ra}_{2}^{(c)}$ for different values of frequency $\omega$ and Vadasz number Va

the eigenvalue of the second-order system is the correction in the critical Rayleigh number, which is obtained by applying the Fredholm alternative condition. The actual Rayleigh number is thus given by (Eq. 31)

$\mathrm{Ra}_{\mathrm{c}}=\mathrm{Ra}_{\mathrm{c}}^{(0)}+\epsilon^{2} \mathrm{Ra}_{\mathrm{c}}^{(2)}$

Noting that $\mathrm{Ra}_{\mathrm{c}}^{(0)}=4 \pi^{2}$ and $\epsilon^{2}>0$, we might now explore the possibility of having subcritical or supercritical motions in the modulated problem depending on whether $\mathrm{Ra}_{\mathrm{c}}^{(2)}$ is negative or positive. This is the prime objective of the paper, in addition to adopting a simpler method than that of Venezian [5] for obtaining the correction Rayleigh number.

Venezian [5] presented results on the temperature-modulated convection problem using plots of $\operatorname{Ra}_{\mathrm{c}}^{(2)}$ versus $\omega$. The present paper reveals two new aspects of the modulation problem outlined in what follows:

- The phase difference between the boundary temperatures rather than the frequency of modulated temperatures determines the nature of influence of modulation upon onset of convection.

- The phase difference may be effectively used as a nonobtrusive external mechanism for regulating the onset of convection.

In view of the foregoing observations, the plot of $\operatorname{Ra}_{\mathrm{c}}^{(2)}$ versus $\theta$ is presented in Fig. 2 for different values of $\omega$ and Va. It is clear from the figure that only for small-modulation frequencies $\mathrm{Ra}_{\mathrm{c}}^{(2)}$ can be positive, and invariably for large frequencies $\mathrm{Ra}_{\mathrm{c}}^{(2)}$ is negative. One other aspect evident from Fig. 2 is that the maximum value of $\mathrm{Ra}_{\mathrm{c}}^{(2)}$ is at a value of $\theta \approx \pi$. We may draw the following conclusions: 

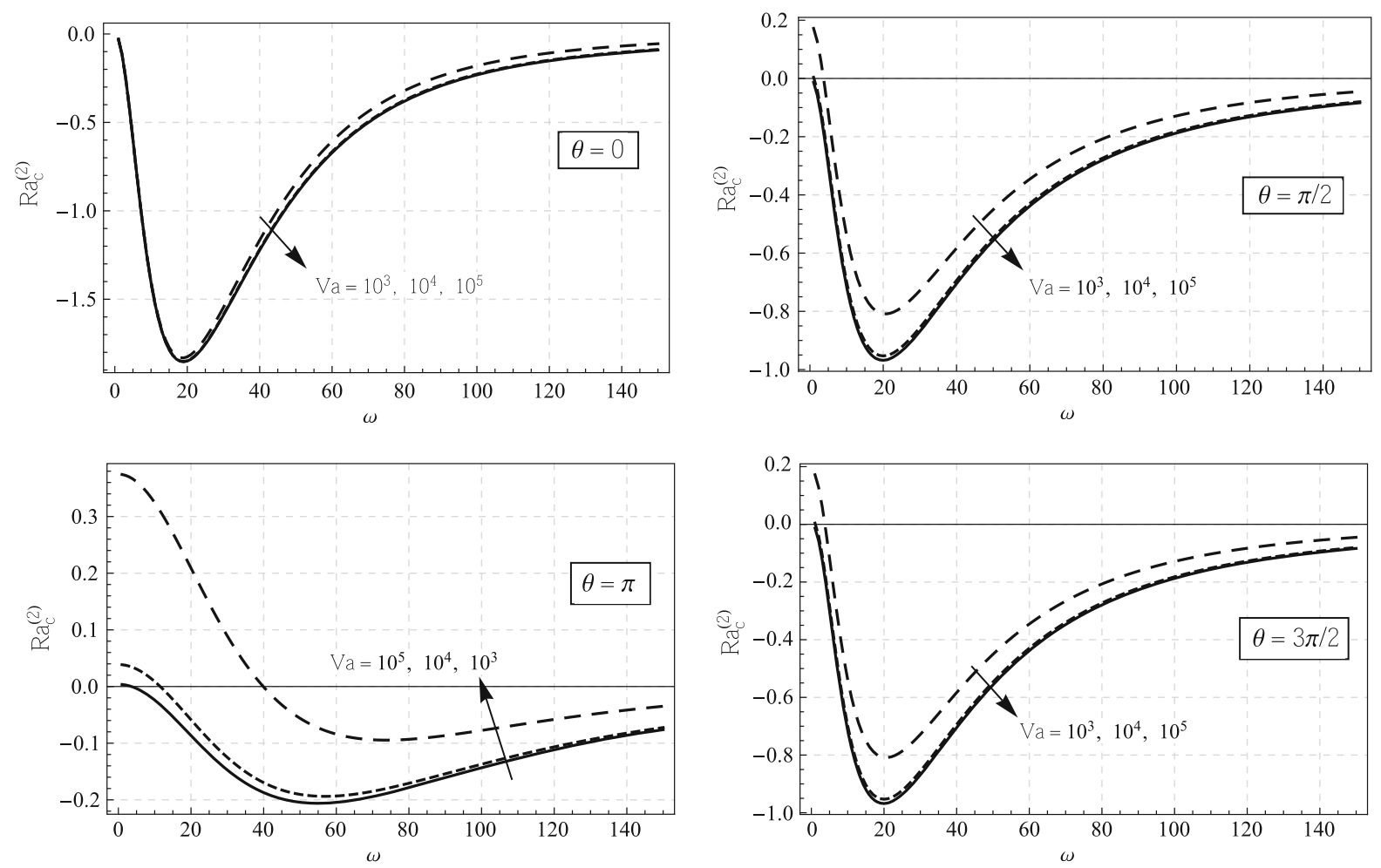

Fig. 3 Effect of $\omega$ on $\mathrm{Ra}_{2}^{(c)}$ for different values of $\theta$ and Va

- In $[0, \pi] \mathrm{Ra}_{\mathrm{c}}^{(2)}$ increases with increases in $\theta$.

- In $[\pi, 2 \pi] \mathrm{Ra}_{\mathrm{c}}^{(2)}$ decreases with increases in $\theta$.

In successive intervals of length $2 \pi$ the preceding results are true.

The following useful results are also discernible from the plots:

- $\mathrm{Ra}_{\mathrm{c}}^{(2)}$ becomes independent of $\mathrm{Va}$ in the range $\mathrm{Va}>10^{4}$.

- The $\mathrm{Ra}_{\mathrm{c}}^{(2)}$ curve gets flatter and flatter as $\omega$ increases. This is in keeping with the observation of Venezian [5] that the modulation effect on the onset of convection vanishes for very large frequencies.

Figure 3 is the conventional Venezian plot of $\operatorname{Ra}_{c}^{(2)}$ versus $\omega$ and is reported in the present paper as a reiteration of the results of Venezian [5]. In particular, the results are depicted for values $\theta=0$ and $\theta=\pi$, which Venezian [5] considered as in-phase and out-of-phase modulations, respectively. The observation made from Fig. 2 holds for the plots of Fig. 3 as well.

Since the porous layer is assumed to be dense, we consider moderate or large values of the Vadasz number. From Figs. 2 and 3 it can be observed that for Va $>10^{4}$ the Vadasz number does not affect the onset of modulated convection significantly. Here we recall the fact that the Vadasz number appears in the governing equations owing to the inclusion of the local acceleration term in the momentum equation. Thus, it can be concluded that one need not include the local acceleration term in a modulated problem involving densely packed porous media.

In summary, we can say that when a time-dependent boundary temperature is present in a problem, it either adds to the damping or deducts from the damping, depending on the type of temperature modulation considered. This is quite evident in the plots of the correction Rayleigh number $\mathrm{Ra}_{\mathrm{c}}^{(2)}$ versus the frequency of temperature modulation shown in Fig. 2.

We now compare the harmonic $(q=\omega)$ and subharmonic $(q=\omega / 2)$ responses of the system to the modulated forcing of frequency $\omega$. For this the effect of various parameters on $\mathrm{Ra}_{\mathrm{c}}^{(2)}$ is depicted in Figs. 4 and 5 for $\mathrm{Va}=1000$. It 

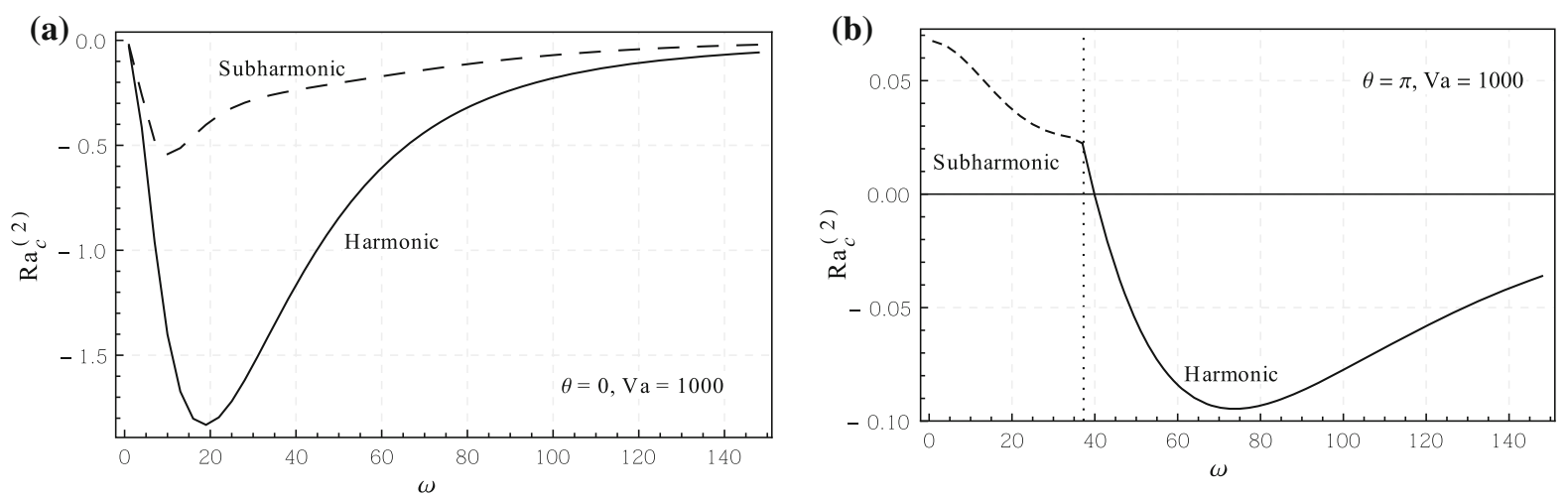

Fig. 4 Transition from harmonic to subharmonic instability with change in $\omega$ for $\mathbf{a}$ in-phase $(\theta=0)$ and $\mathbf{b}$ out-of-phase $(\theta=\pi)$ modulation
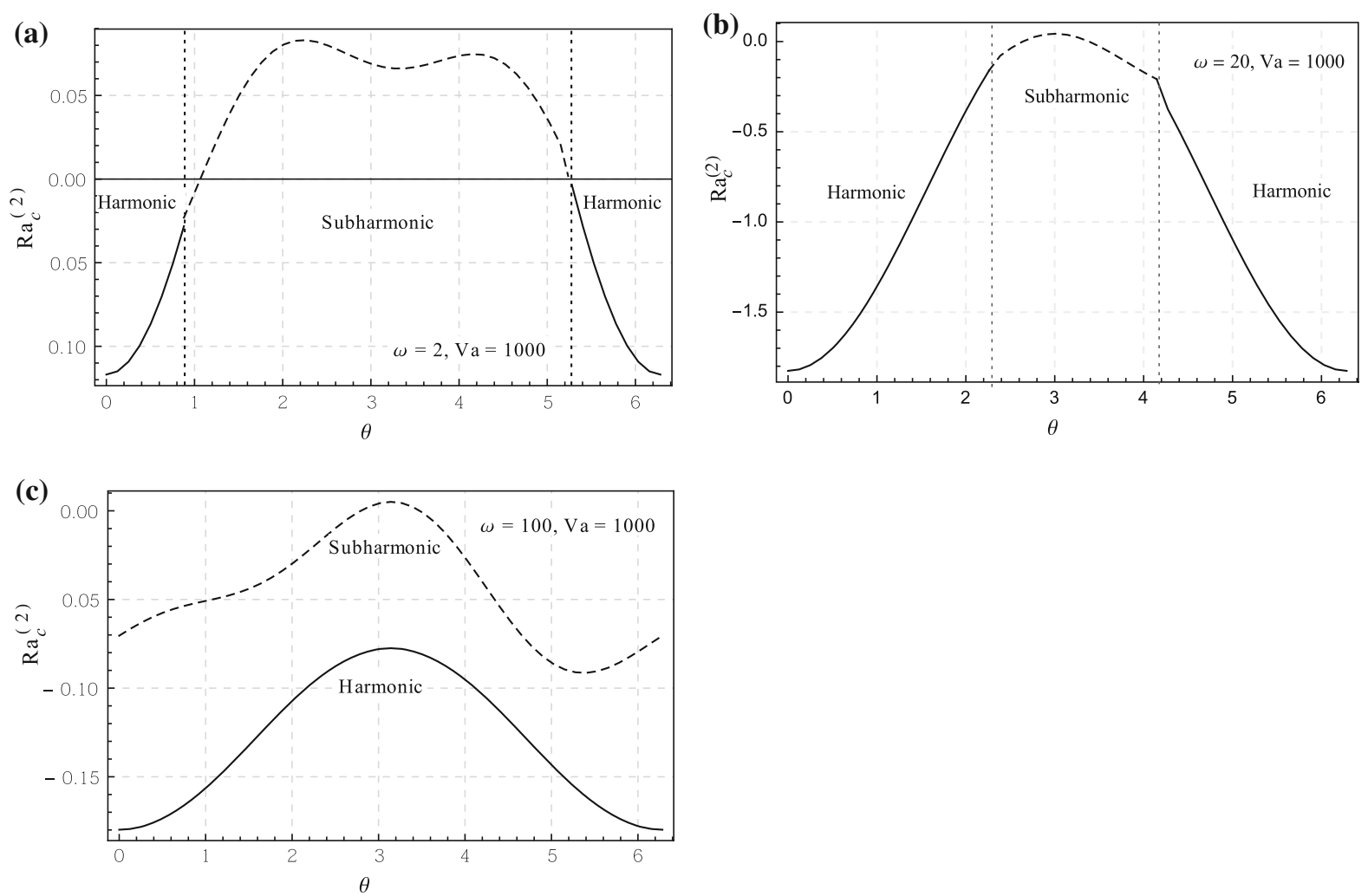

Fig. 5 Transition from harmonic to subharmonic instability with change in $\theta$ for $\mathbf{a} \omega=2, \mathbf{b} \omega=20$, and $\mathbf{c} \omega=100$

can be observed that in the case of in-phase temperature modulation, harmonic stability is preferred over subharmonic stability as $\left.\mathrm{Ra}_{\mathrm{c}}^{(2)}\right|_{q=\omega}<\left.\mathrm{Ra}_{\mathrm{c}}^{(2)}\right|_{q=\omega / 2}$ for all values of the modulation frequency (Fig. 4a). In the case of out-of-phase modulation the subharmonic instability appears for small values of the modulation frequency $(\omega<38$ approx.), and for moderate and large values the harmonic instability persists (Fig. 4b). $\left(\mathrm{Ra}_{\mathrm{c}}^{(2)}, \theta\right)$ curves are plotted for different frequencies in Fig. 5. From these figures it can observed that there exists an interval $\left[\theta_{1}, \theta_{\mathrm{r}}\right]$ in which subharmonic instability appears. Whenever $\theta<\theta_{1}$ or $\theta>\theta_{\mathrm{r}}$, harmonic stability is preferred. It is apparent that these values $\theta_{1}$ and $\theta_{\mathrm{r}}$ depend on the modulation frequency and Vadasz number. Moreover, this interval $\left[\theta_{1}, \theta_{\mathrm{r}}\right]$ decreases as $\omega$ increases, and for sufficiently large values of $\omega$ only harmonic instability can be detected as the preferred mode (Fig. 5c). 


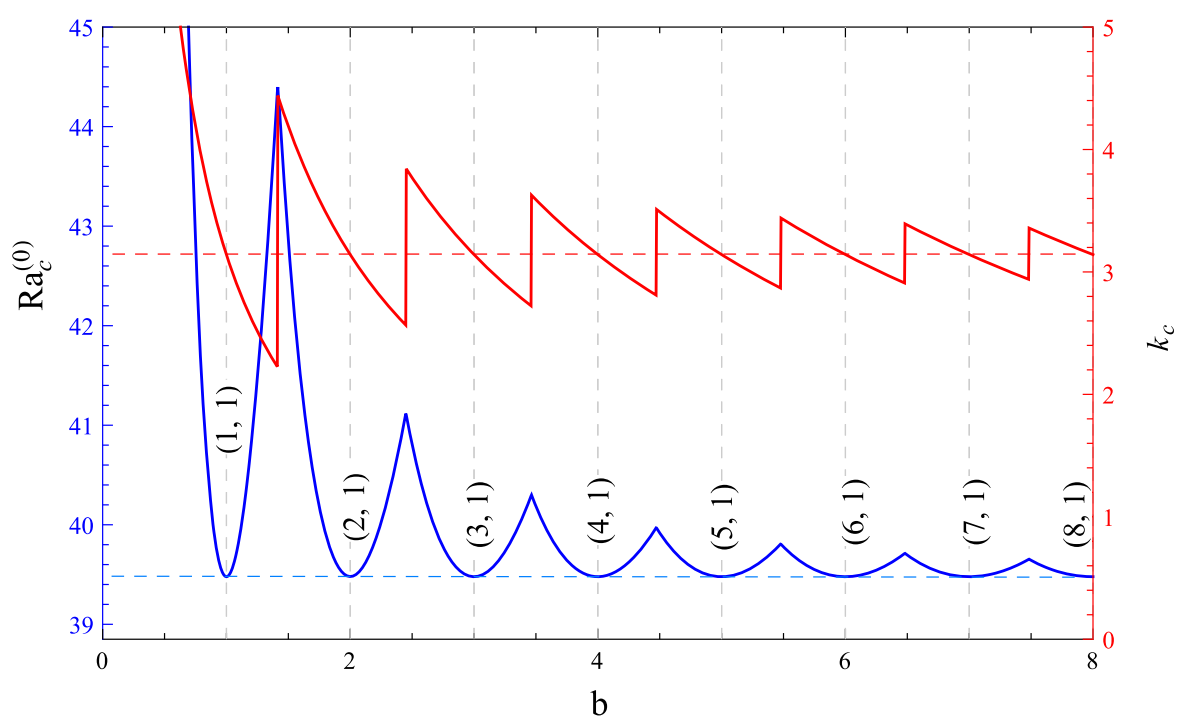

Fig. $6 \mathrm{Ra}_{\mathrm{c}}^{(0)}$ and $k_{\mathrm{c}}$ versus aspect ratio $b$

To make the paper self-contained, we reproduce the plot revealing the relationship between the nondimensional width (aspect ratio) and the critical Rayleigh number $\mathrm{Ra}_{\mathrm{c}}^{(0)}$ as Fig. 6 . We note here that the minimum value of the Rayleigh number is $4 \pi^{2}$ in the case of integer values of $b$, and in these cases $n=b$ (see Tyvand [16, Chap. 4]), which is a reiteration of the results we obtained for Darcy-Bénard convection. Thus, in our analysis, pertaining to the modulated convection in a rectangular porous enclosure, it is mainly the noninteger values of the nondimensional width $b$ that are of interest. Figure 6 delineates the fact that the critical Rayleigh number depends on the aspect ratio and, hence, on the number of cells. This is because of the fact that the number of cells in an enclosure crucially depends on the aspect ratio. As noted by Tyvand [16, Chap. 4], integer values of $b$ yield the same result as that of Darcy-Bénard convection. This result is thus true for both modulated and nonmodulated situations. The plot of $k_{\mathrm{c}}$ in this figure reiterates our earlier comments on the relation between number of cells in an enclosure and aspect ratio.

One should note here that on substituting these results related to convection in a porous enclosure in the results concerning the modulated Darcy-Bénard convection of the previous section, we can obtain the results of the modulated porous enclosure problem. The correction in the critical Rayleigh number $\operatorname{Ra}_{\mathrm{c}}^{(2)}$ is evaluated from Eq. (39) using the value of $\mathrm{Ra}_{\mathrm{c}}^{(0)}$ given in Eq. (44). We first discuss the results pertaining to $\mathrm{Ra}_{\mathrm{c}}^{(2)}$ in the case of harmonic responses $(q=\omega)$. The comparison of harmonic and subharmonic responses will be taken up later.

The critical Rayleigh number of the thermally modulated problem depends on the critical Rayleigh number of the problem without modulation, $\mathrm{Ra}_{\mathrm{c}}^{(2)}$, the small amplitude of modulation $\epsilon$, and a correction Rayleigh number. The plot of $\mathrm{Ra}_{\mathrm{c}}^{(0)}$ versus $b$ is given in Fig. 6. The dependence of $\mathrm{Ra}_{\mathrm{c}}^{(2)}$ on $b$ is shown in Fig. 7 for different frequencies and different values of the Vadasz number. For small as well as large frequencies we find that there can be only subcritical motion when there is no phase difference between the boundary temperatures. However, when there is a phase difference, both supercritical and subcritical motions are possible. In this case, for small frequencies we can have supercritical motions, and for large frequencies we can have subcritical motions. Our computations reveal that for small amplitudes and very large frequencies of modulation the results of the thermally modulated and nonmodulated problems are the same. Figure 7 also reveals a result on the Va influence on the correction Rayleigh number similar to that of the problem with modulation. We found that for $\mathrm{Va}>10^{4}$ the correction Rayleigh number becomes independent of $\mathrm{Va}$, and in this case the motion is invariably subcritical.

The conclusions of the present problem are given below. 

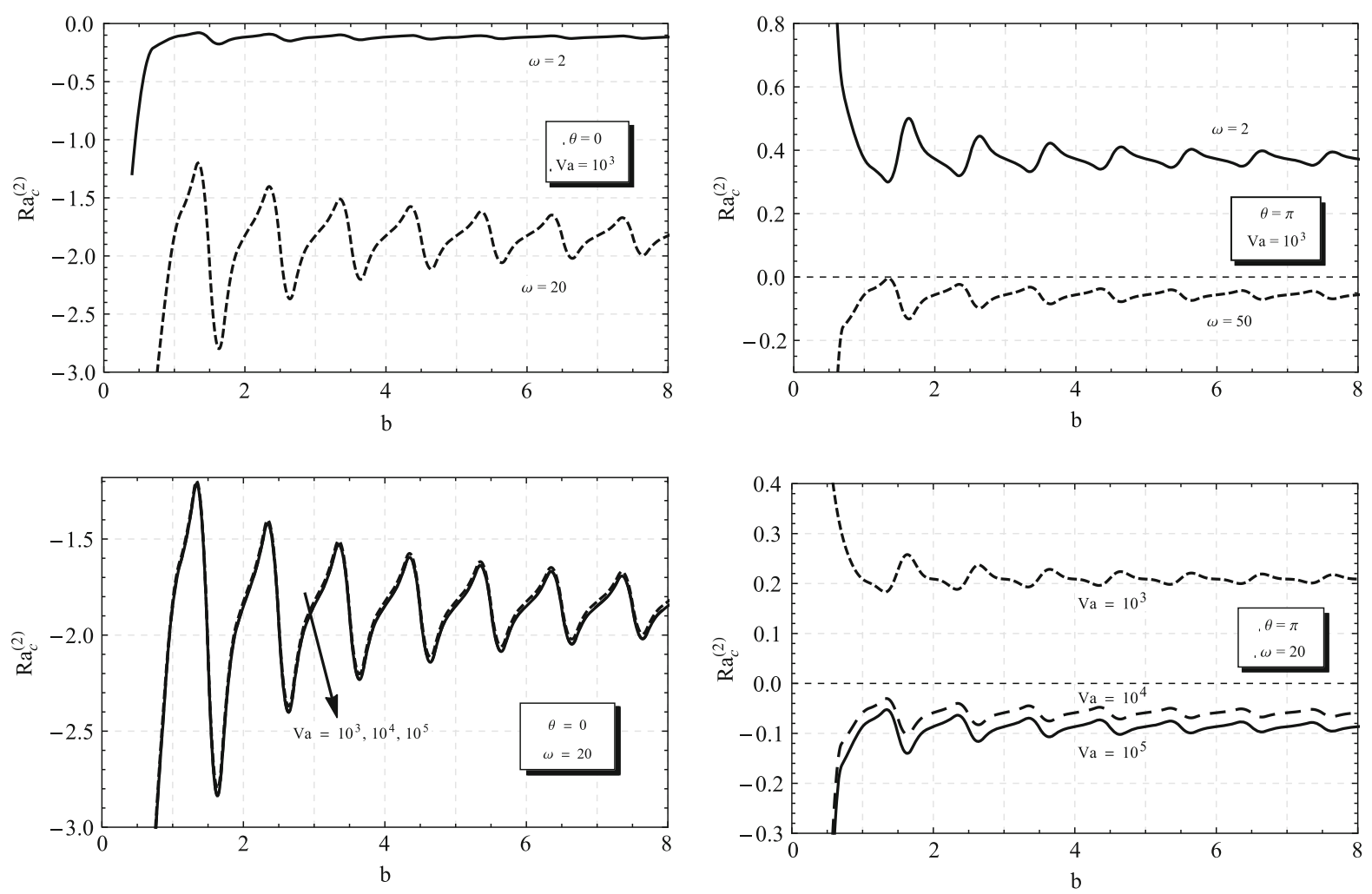

Fig. $7 \mathrm{Ra}_{\mathrm{c}}^{(2)}$ for porous enclosure problems for different values of parameters

\section{Conclusions}

1. For moderate values of the modulation frequency, we obtain the following results:

(a) $\left[\mathrm{Ra}_{\mathrm{c}}\right]_{\epsilon=0}>\left[\mathrm{Ra}_{\mathrm{c}}\right]_{\epsilon \neq 0}$ for synchronous temperature modulation.

(b) $\left[\mathrm{Ra}_{\mathrm{c}}\right]_{\epsilon=0}<\left[\mathrm{Ra}_{\mathrm{c}}\right]_{\epsilon \neq 0}$ or $\left[\mathrm{Ra}_{\mathrm{c}}\right]_{\epsilon=0}>\left[\mathrm{Ra}_{\mathrm{c}}\right]_{\epsilon \neq 0}$ for asynchronous temperature modulation, depending on the choice of $\omega$ and $\theta$.

2. The local acceleration term can also be neglected for the convective flows in densely packed porous media in the case of the modulated problem.

3. Subharmonic instability is preferred only for a certain range of values of phase angle and modulation frequency. In the case where (a) $\theta \ll 1$ (for all values of $\omega$ ) and (b) $\omega \gg 1$ (for all values of $\theta$ ), the harmonic solution yields the critical mode of convection.

4. The results of the corresponding modulation problem in a rectangular porous enclosure (RPE) can be obtained from the results of the Darcy-Bénard convection (DBC) problem. Further, we find that:

(a) $\left[\mathrm{Ra}_{\mathrm{c}}^{(0)}\right]_{\mathrm{DBC}}=\left[\mathrm{Ra}_{\mathrm{c}}^{(0)}\right]_{\mathrm{RPE}}$ and $\left|\left[\mathrm{Ra}_{\mathrm{c}}^{(2)}\right]_{\mathrm{DBC}}\right|=\left|\left[\mathrm{Ra}_{\mathrm{c}}^{(2)}\right]_{\mathrm{RPE}}\right|$ for integer values of $b$;

(b) For noninteger values of $b$ the critical values of the two cases are unequal.

5. The present method of obtaining the critical Rayleigh number for a modulated problem with small-amplitude modulation can be very easily used in convection problems with any type of modulation mechanism or any boundary combination, and this is the novelty of the method.

Acknowledgments The authors are grateful to the three referees and the editor whose instructive comments helped us to refine our paper. 


\section{References}

1. Horton CW, Rogers FT (1945) Convective currents in a porous medium. J Appl Phys 16:367-370

2. Lapwood ER (1948) Convective of a fluid in a porous medium. Proc Camb Philos Soc 44:508-521

3. Katto Y, Masuoka T (1967) Criterion for the onset of convective flow in a fluid in a porous medium. Int J Heat Mass Transf 10:297-309

4. Kladias N, Prasad V (1988) Natural convection in a horizontal porous layer: effect of Darcy and Prandtl numbers. In: Proceedings of the National Heat Transfer Conference, ASME HTD 96, pp 593-604

5. Venezian G (1969) Effect of modulation on the onset of thermal convection. J Fluid Mech 35:243-254

6. Malashetty MS, Wadi VS (1999) Rayleigh-Bénard convection subject to time dependent wall temperature in a fluid saturated porous layer. Fluid Dyn Res 24:293-308

7. Chhuon B, Caltagirone JP (1979) Stability of a horizontal porous layer with time-wise periodic boundary conditions. ASME J Heat Transf 101:244-248

8. Bhadauria BS (2007) Thermal modulation of Rayleigh-Bénard convection in a sparsely packed porous medium. J Porous Media 10(2):175-188

9. Bhadauria BS, Suthar OP (2009) Effect of thermal modulation on the onset of centrifugally driven convection in a vertical rotating porous layer placed far away from the axis of rotation. J Porous Media 12(3):239-252

10. Malashetty MS, Basavaraja D (2002) Rayleigh-Bénard convection subject to time-dependent wall temperature/gravity in a fluid saturated anisotropic porous medium. Heat Mass Transf 38:551-563

11. Malashetty MS, Basavaraja D (2003) The effect of thermal/gravity modulation on the onset of convection in a horizontal anisotropic porous layer. Appl Mech Eng 8(3):425-439

12. Niemela JJ, Donnelly RJ (1987) External modulation of Rayleigh-Bénard convection. Phys Rev Lett 59:2431-2434

13. Govender S (2004) Stability of convection in a gravity modulated porous layer heated from below. Transp Porous Media 57:113-123

14. Beck JL (1972) Convection in a box of porous material saturated with fluid. Phys Fluids 15(8):1377-1383

15. Nield DA, Bejan A (2006) Convection in porous media. Springer, New York

16. Tyvand PA (2002) Onset of Rayleigh-Bénard convection in porous bodies. In: Ingham DB, Pop IA (eds) Transport phenomenon in porous media-II. Pergamon, London

17. Raju VRK, Bhattacharya SN (2010) Onset of thermal instability in a horizontal layer of fluid with modulated boundary temperatures. J Eng Math 66:343-351

18. Siddheshwar PG (2010) A series of solution for the Ginzburg-Landau equation with time-periodic coefficient. Appl Math 1(6):542554

19. Jordan DW, Smith P (2007) Nonlinear ordinary differential equations: an introduction for scientists and engineers, 4th edn. Oxford University Press, New York

20. Bhattacharjee JK (1987) Convection and chaos in fluids. World Scientific Press, Singapore

21. Siddheshwar PG, Vanishree RK, Melson AC (2012) Study of heat transport in Bénard-Darcy convection with g-jitter and thermomechanical anisotropy in variable viscosity liquids. Transp Porous Media 92(2):277-288

22. Siddheshwar PG, Bhadauria BS, Suthar OP (2013) Synchronous and asynchronous boundary temperature modulations of BénardDarcy convection. Int J Non Linear Mech 49:84-89 